\title{
MDMC : A WSN cooperative protocol for Minimizing the Data Distortion
}

\author{
Floriano De Rango*, Amilcare Francesco Santamaria ${ }^{\dagger}$, Peppino Fazio ${ }^{\ddagger}$, and Pierfrancesco Raimondo ${ }^{\S}$ \\ University of Calabria \\ Rende, Italy \\ Email: $\left\{{ }^{*}\right.$ derango, ${ }^{\dagger}$ afsantamaria, ${ }^{\ddagger}$ pfazio, ${ }^{\S}$ p.raimondo $\} @$ deis.unical.it
}

\begin{abstract}
In this work a new protocol able to obtain network cooperation in a wireless sensor network reducing the control overhead and reducing the data distortion is developed. Its aim is to select the minimum number of sensors able to cover the phenomena and to report not correlated data to the sink in order to reduce distortion. The proposed protocol is called Minimal Distortion with Maximal Cover (MDMC) protocol and it tries, on the one hand, to gradually expand the covered phenomenon area and, on the other hand to reduce the distortion that arises from the interaction of the sensors. To reach this goal, the protocol uses two heuristics along with an activation technique, the first ones select the sensors on the basis of their features, whereas the second one decreases the number of sensors which take part in the coalition formation process. The proposed has been compared with a cluster based protocol such as Local Negotiated Clustering Algorithm (LNCA).
\end{abstract}

Keywords-WSN, Clustering, Heuristics, Coalition

\section{INTRODUCTION}

The aim of this work is to define and to develop a new cooperative protocol in a wireless sensor network able to promote the coalition constitution of sensor nodes that are more representative in the sensor network. The motivations of this contribution are due to the limited energy resources and low computational and transmission capacities of sensor nodes that impose the selection of best candidates nodes without involving all nodes in the report generation and forwarding phases. Considering that the data sensed by sensors are not all important at the same time due to the coverage of the same portion of phenomena by the same sensor nodes, sometimes it can be more useful to exploit the concept of spatial correlation in order to select the most appropriate nodes able to generate not correlated data and able to provide more info at the sink about the monitored phenomena [1]. The capacity to select fewer sensor nodes distributed on the coverage area allows the reduction of the number report packets offering reliable information about the phenomena and reducing the distortion at the same time. This contribution tries to combine different aspects which are sometimes faced separately in proposed protocols for Wireless Sensors Network (WSN). In particular, the proposed protocol is based on the coalition process that is regulated by two specific metrics: coverage area and distance between sensor nodes and phenomena. These two metrics have been joined using a utility function that regulates the building of a coalition of the most representative nodes. A cluster based protocol called LNCA has been considered for comparison purpose. It selects the sensor nodes on the basis of the similarity of the sensed data. Our approach is able to reduce the distortion calculated at the sink preserving energy saving and reducing the control overhead in comparison with LNCA. The protocol selects a sensor subset, among those activated by the activity of the phenomenon. The members of this subset will send their own reports to the sink ensuring a reliable measurement of the phenomenon at the sink. The coalition process scope is to cover the whole phenomenon decreasing the distortion that is brought about by sensor interaction as much as possible. By using heuristics, the protocol MDMC is able to reduce the number of sensors that report the information to the sink without worsening the reliability of the latter. The heuristics drive the selection evaluating the feature of each sensor and its contribution from the coalition viewpoint. The rest of the paper is organized as follows: In Section 2, the related works in which we deal with the concepts just discussed are reported. In section 3 a formal presentation of the protocol MDMC is provided; In section 4 the Activation Probability mechanism is presented; In section 5 , instead, the algorithm for coalition process is described. In summary, in section 6 our simulation results are shown and this paper is concluded in section 7 .

\section{RELATED WORKS}

Many clustering protocols for WSN have been proposed in literature [2]. Each of them tries to optimize some parameters such as energy spent, control overhead, network lifetime etc . An interesting study is proposed in [3], [4] in which network issues and protocol evaluation are analyzied for approaching Green communication and Energy Harvesting. Different approaches and models are used to build clusters; in [5], for example, authors propose a genetic based algorithm to build clusters, although, in [6] authors propose a k-means protocol to build cluster of sensors. Moreover, It is possible to find random clustering techniques, based on k-hop clustering [7], location-based clustering [8], centralized clustering [9], data aggregation and clustering,Quality of Service (QoS) oriented clustering [10]. In this contribution, our proposal is compared with a LNCA [7], a clustering protocol belonging to the family of k-hop clustering, where the size and the shape of the clusters is related to the similarity of the sensed data. We chose it 
as the comparison, because it is the most similar with the concept of distortion and correlated data that we adopted in the proposed metrics. In the [11] the authors involve in the base station in the cluster formation. They start form the hypothesis that the method of single-hop data transmission increases the energy burden of the nodes. Thus they use the K-means++ algorithm to divide the cluster evenly in the base station and at the end of the procedure the shortest path is selected for inter-cluster transmission in the stage of data transmission. In the WSN, clustering can enhance overall system scalability and energy efficiency; in [14] a Distributed Cluster Head Scheduling (DCHS) algorithm is proposed to increase the network longevity by satisfies an ideal distribution of the cluster head among the sensor nodes and avoids frequent selection of cluster head, based on Received Signal Strength Indication (RSSI) and residual energy level. In the [15] the authors propose an energy efficient clustering algorithm. The optimum one-hop distance and clustering angle are formulated by minimizing the energy consumption between inter-cluster and intra-cluster. They demonstrated that with the proposed mechanism, the frequency of updating cluster head and the energy consumption for establishing new cluster head can be reduced. Even more, taking into account energy saving another technique has been proposed by author in [16]. Here the solution is based on changing dynamically and with more efficiency the Cluster Head $(\mathrm{CH})$ election probability.

On the basis of these approaches, we considered in our coalition process a procedure that inherits some aspects of the survey proposed in [12]. Using the concept of a cooperation graph we model the coalition formation process to find a stable coalition that guarantees a reliable report about the activity of the phenomenon. In order to achieve this aim, the Connected Dominating Set (CDS)s building technique is used to propagate the information inside the network. Moreover, we defined two metrics that are applied with an activation probability to affect the coalition process dynamic. The coalition process is distributed and inherits some behaviors of the minimum CDSs as referred to [12]. Then, a distributed protocol is developed by forming a coalition and reporting phenomenon measurements to the sink. In order to show the effectiveness of our proposal, the MDMC. protocol has been compared with LNCA in terms of control overhead, distortion at the sink, energy spent and temperature of the phenomena computed at the sink. In brief, the main contributions of our work are summarized below:

- A definition of two heuristics: one based on the sensor cover area and the second stated by utility function. These heuristics drive the coalition process among sensors.

- An activation probability has been defined to re- duce the number of potential sensors involved in the process coalition.

- A distributed and dynamic coalition process has been proposed.

- Performance evaluation of the MDMC protocol compared with a previous work LNCA has been presented considering many performance metrics such as distortion, consumed energy, number of control packets.

\section{MDMC PROTOCOL}

We consider a wireless sensor network with $n$ sensors, in some areas. Each sensor is equipped with an omni directional antenna with communication range $R_{c}$, and with an application-specific sensing device, with range $R_{s}$. To ensure connectivity among sensors we impose $R_{c}=2 * R_{s}$. We assume that a physical phenomenon propagates in every direction upon an area where $m$ of $n$ sensors are deployed. We define the neighbors of the sensor $s_{i}$ as the sensors set that is inside the communication range of sensor $s_{i}$, and let us indicate it with $N\left(s_{i}\right)$. Our goal is to select a subset of these $\mathrm{m}$ sensors that will send their own reports to the sink. An important aspect of the distributed environment, such as the sensor networks, is the high cost that involves any cooperation process. The process cost increments progressively with the increase in the number of participants. The cooperation entails a high energy consumption since those networks are formed by hundreds or thousands of sensors. Using a selection criterion to narrow the participating process, it is possible to improve both the reliability and longevity of the network. A deep analysis of our problem has determined the choice of two heuristic principles: spatial correlation and covered phenomenon value. In addition to these heuristics we also use an activation probability to reduce the number of potential cooperating sensors. Following this section our reasons for these choices are provided.

\section{A. Utility function}

Here, we introduce the first of the two heuristics as a utility function to model the sensor contribution in the coalition. This function is able to evaluate the positive contribution on the phenomenon observation and the negative one grounded on the distortion function defined in (1). This idea arises from the intuition that selecting sensors that are distant enough from one another is better than choosing a close sensor set, since close sensors would be subject to the same interference which alters their measurements. The authors in [1] define a framework to study the spatial correlation from a report set coming from wireless sensor networks. Using $M$ packets received at the sink about the activity of the phenomenon $F$, they compute the distortion value as:

$$
\begin{aligned}
& \left.D(m)=\sigma F^{2}-\frac{\sigma F^{4}}{M\left(\sigma F^{2}+\sigma N^{2}\right)}\left(2 \sum_{i=1}^{M}\right) \rho_{(s, i)}-1\right)+ \\
& \frac{\sigma F^{6}}{M^{2}\left(\sigma F^{2}+\sigma N^{2}\right)^{2}} \sum_{i=1}^{M} \sum_{j \neq i}^{M} \rho_{(i, j)}
\end{aligned}
$$

where:

- correlation coefficient $\rho_{(s, i)}$ : distortion grows along with the distance between the sensor and phenomenon source. It is evident that far sensors report less accurate measurements compared to closer ones.

- correlation coefficient $\rho_{(i, j)}$ :this coefficient penalizes the reports that come from sensors which are too close to one another. 
Both $\rho_{(s, i)}$ and $\rho_{(i, j)}$ are easy to compute using correlation models among those present in literature. Without loss of generality in this work we have chosen the spheric one, defined in [13], this is a popular model with the distinctive feature that any two observations taken more than $\theta_{1}$ distance units apart are uncorrelated :

$$
K_{\theta}(d)= \begin{cases}1-\frac{3}{2} \frac{d}{\theta_{1}}+\frac{1}{2}\left(\frac{d}{\theta_{1}}\right)^{3}, & \text { if } 0 \leq d \leq \theta_{1} \\ 0, & \text { if } d \geq \theta_{1}\end{cases}
$$

Now, we can state the utility function on a sensor set $C$ as :

$$
F(C)=\lambda * B-\mu * M .
$$

where $\lambda$ and $\mu$ are weights used to emphasize the positive and negative terms; the terms $B$ and $M$ are defined as:

$$
\begin{gathered}
B=|C| *\left(2 \sum_{i \in C} \rho_{(s, i)}-1\right) \\
\left.M=\sum_{i \in C} \sum j \neq i, j \in C \rho_{(} i, j\right)
\end{gathered}
$$

Both $B$ and $M$ are stated from (1) isolating the positive effect (increment) and the negative one (decrease) given to function (3) .Then the necessary condition in order that a couple of coalitions $C_{1}$ and $C_{2}$ could join and build a third one, namely $C_{3}=C_{1} C_{2}$, is:

$$
\begin{aligned}
& \lambda * B_{C_{1}}-\mu * M_{C_{1} \cup C_{2}} \geq 0 \wedge \\
& \wedge \lambda * B_{C_{2}}-\mu * M_{C_{1} \cup C_{2}} \geq 0
\end{aligned}
$$

Holding of the condition (6) guarantees that $F\left(C_{3}\right) \geq$ $F\left(C_{1}\right)+F\left(C_{2}\right)$

\section{B. Covered phenomenon value}

The heuristic based on distortion alone is not sufficient enough to ensure a uniform cover of the whole phenomenon. So it was necessary to introduce a heuristic that gave prominence to the relative sensors position and the size of their covered segments. It is assumed that the phenomenon propagation proceeds with a linear trend without deformation, and the sensing area is fixed for each sensor and its shape can be assimilated with a disk. A set of sensors $S=S_{1}, \ldots, S_{n}$ deployed in a bi-dimensional area $A$ are considered. The sensing range is fixed to $R_{S}$, now for each sensor $i$ naming its coordinate $\left(x_{i}, y_{i}\right)$ in $A$, we can claim the following definitions:

- Definition 1: A phenomenon in $A$ is said to be wholly covered by $s_{i}$ if it is inside the $s_{i}$ sensing area. Instead a phenomenon is said to be $j$-covered if it is inside the sensing areas of at least the $j$ sensors.

- Definition 2: Given a natural number $k$, the problem of $k$-covered phenomenon is a decision problem, and its aim is to find out if all points of the phenomenon in $A$ are $k$-covered or not. We have focused on the perimeter of the phenomenon propagation area and how

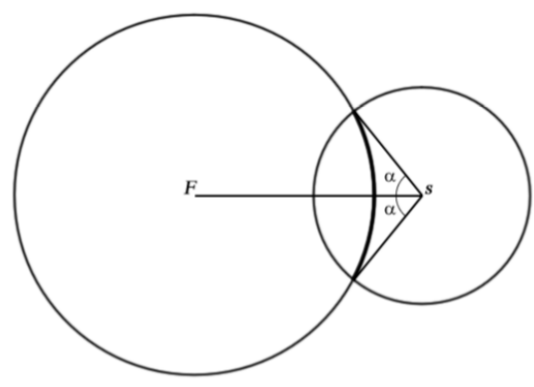

Fig. 1. Perimeter segment of $F$ covered by s. In this case the sensor s covers the interval $[(2 \pi-\alpha), \alpha]$.

this perimeter is covered by sensors. For example, a sensor $s_{i}$ and the phenomenon $F$ are localized respectively at $\left(x_{i}, y_{i}\right)$ and $\left(x_{F}, y_{F}\right)$. Their distance is $d\left(F, s_{i}\right)=\sqrt{\left|x_{F}-x_{i}\right|^{2}+\left|y_{F}-y_{i}\right|^{2}}$. The sensor senses the phenomenon if it is inside the propagation area that is $d\left(F, s_{i}\right) \leq R_{F}$ The sensors covering is expressed by:

- angle $\theta$ : with the size of this angle we are able to collocate the sensor upon a circumference that has the phenomenon as center. So we have to normalize the sensors coordinate in the phenomenon-center system, $\left(\hat{x}_{i}, \hat{y}_{i}\right)$, and so :

$$
\theta=\arcsin \left(\frac{\hat{y}_{i}}{{\sqrt{\hat{x}_{i}^{2}+\hat{y}_{i}}}^{2}}\right)
$$

- size $\alpha$ : to compute it we have to know the distance between sensor and phenomenon $d(F, s)$, the sensing range $R_{s}$ and the propagation range $r_{F}$. With these values we have:

$$
\alpha=\arccos \left(\frac{2 d\left(F, s_{i}\right)^{2}-R_{s}^{2}}{2 d\left(F, s_{i}\right)^{2}}\right)
$$

An illustration is shown in Fig.1. Now we are going to use the cover concept defined above, but first we have to give the following definition:

- Definition 3: Given a sensor $s_{i}$, its cover $c_{i}$ is expressed by the interval $[(\theta-\alpha),(\theta+\alpha)]$. To store the segments of the covered phenomenon, we use a vector defined into $[0,2 \pi]$, this represents the phenomenon perimeter and so it is easy to evaluate the covering of whole phenomenon. Using the covered phenomenon value we can define the concept of independent uses in the graph theory with a concept closer to our scope to model the second heuristic;

- Definition 4: Given two sensors $s_{i}$ and $s_{j}$, they are independent if their own cover areas are totally disjointed, that is no overlapping. Considering the high density of wireless sensor net work to use a heuristic based on the independence among sensors could be difficult with the definition just stated above. So it will relax by opportunely using a overlapping(AOL) threshold defined as $A O L=2 \alpha * x$ where $\alpha$ is defined in (8) and $x$ is the threshold expressed in percentage; 
- Definition 5: Given two sensors $s_{i}$ and $s_{j}$, they have weak-independence if their overlapping value is less than the allowed overlapping threshold. Again we have found another necessary condition using the allowed overlapping threshold. This choice gives more flexibility to our protocol, enabling a more accurate conformation varying network topology.

\section{ACTIVATION PROBABILITY}

Activation probability is grounded on the distance from the phenomenon and the sensors remaining energy resource. The value of this probability is used to delay the sensor selection correspondingly with its features in the coalition formation process. For example, the higher the value of probability it will be more probable that the sensor will be selected as potential member. We assume the sensors are able to localize themselves and the phenomenon source, and so compute their distance from it. Naming $A$ the interest area, in which $n$ sensors $S=S_{1}, \ldots, S_{n}$ are randomly deployed, their location set $L=L_{1}, \ldots, L_{n}$ and their energy resources, $r_{k} \in[0,1]$ being known, we can write the activation probability at time $t$ as:

$$
p_{A}(t)= \begin{cases}{\left[r_{k}\right]^{\alpha} *\left[\frac{F_{k}-\left\|L_{k}-l_{o}(t)\right\|}{F_{k}}\right]^{\beta},} & \text { if }\left\|L_{k}-l_{o}(t) \leq F_{k}\right\| \\ 0, & \text { otherwise }\end{cases}
$$

Where $l_{o}(t)$ represents the phenomenon position at time $t$, and $F_{k}$ is the area covered by sensor $s_{k}$. Since both the terms are in $[0,1], \alpha$ and $\beta$ may be used to highlight the relevance of the two features. Using this probability value a leader delays sensor selection, and it follows participation in the coalition formation process. The rationale of the proposed activation probability is the following: giving more chances to be part of the starting coalition process to sensor nodes that present more residual energy (energy resources $r_{k}$ ) or to sensor nodes that are closer to the phenomenon producing a lower distortion value. Obviously, not all sensors close to the phenomenon should be stressed in order to avoid creating network partition and for this reason the energy resource represented by $r_{k}$ can be useful.

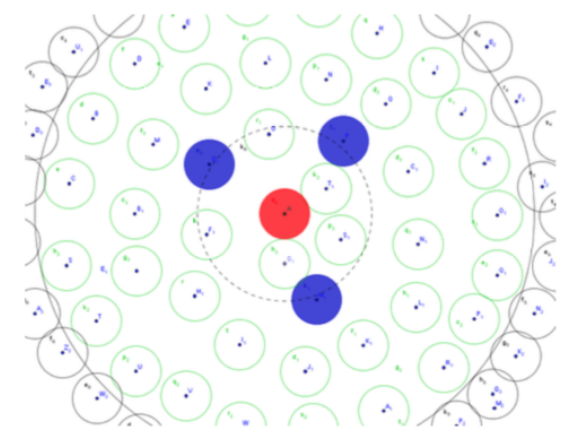

Fig. 2. Selected leaders among sensors in the first ring.

\section{Algorithm for CoAlition Process}

In order to avoid a chaotic coalition formation process, we partition the interest area into $k$ concentric disks. Each disk has a radius $R_{L}=k 2 R_{c}$ wherek 1 . The Coalition Formation Process (CFP) begins when sensors sense the phenomenon activity for the first time. Then each sensor delays its entry into CFP using an activation probability. This probability relies on the distance from the phenomenon source and sensor energy resource. When a sensor begins the CFP, it sends its own information to its neighbors at one-hop. After a period $\tau$ each sensor tries to build a coalition with its created neighbor set. Any sensor that can build a coalition with at least two sensors claims it to its neighbors at one hop. The leader is the sensor that has built a coalition with the highest value of effective cover among its neighbors. When a sensor evaluates two sensors, it is going to check that their interaction respects the coalition constraints described in (6). A hypothetical situation that describes such a process is shown in Fig.2. The leader also has to send a message to leaders staying two hops away from it, in order acquire the two- hop information needed to choose the best one. Once all those messages have been received, it is possible to choose the sensor with the best coalition. This one then sends an activation message to its direct neighbors that are member of its coalition, and after that another message to its neighbor leaders at two hops. Receiving the activation message from the leader, sensors become the first selected sensors that will send a report to the sink when the reporting phase starts. In order to reduce the number of sensors taking part to CFP, an active sensor sends a shadow cone message, when a sensor receives this message it evaluates if it is inside the active sensors shadow cone, if it is then it does not participate in the following coalition formation process. In the meanwhile the neighbor leaders, receiving the message about the formed coalition, evaluate their possible extensions using their neighbor sensors. These extensions are sent to the coalition manager that will combine them and choose the next coalition manager. The chosen leaders received the message to take control of the coalition, repeat this process till all the sensors in the first ring are explored and there are no more feasible extensions. When a leader finds another formed coalition and it is the manager of another one, then it will join them in a third one. By partitioning the area and fixing the size of the rings, the effects of these joins never break the coalition constraints. In the second phase, the active sensors continue building the coalition with the sensors which stay into the next ring. Each member sends a message with the information about the inner coalition. This message is only processed by sensors that stay in the current ring, then these sensors store a reference to its parent coalition member relay the message and send information about itself again. We prefer to give a hierarchical order to the process, to ensure an easier and more efficient extension process. The sensors can start to evaluate a possible extension, but now when a sensor finds one it directly sends its extension to its parent member. Once all the extensions have been received, a coalition member 
combines them and sends this new extension to the coalition manager. The coalition manager then tries again to combine all the received extensions to build the coalition as well as possible and so forth in the next rings.

\section{Simulations RESUlts}

In this section simulation results are presented. In order to carry out results we use the Omnet++ [17] with Castalia [18] environment to simulate the sensor network. Low layers are configured with default parameters as proposed by castalia framework. Application layer has been customized to follow our proposal model. For the sake of completeness, we have chosen the CC2420 as the Radio module, TMAC as MAC module and MultipathRingsRouting as routing protocol. We perform our simulations guaranteeing thirty complete processes to form a stable coalition, for each configuration we perform ten different runs. At the end the average values are evaluated.

\section{A. Sensing Range}

In the initial simulations we use a topology of 225 sensors deployed into an area of $200 \times 200 \mathrm{~m}^{2}$ in gridded order of $15 \times 15$ lines. We assume a maximum communication range, $R_{c}=40 \mathrm{~m}$, to all sensors and the propagation of the phenomenon staying at the area center follows a circular shape with radius $R_{F}=100 \mathrm{~m}$. In any simulation run we choose a sensor as sink with a fixed position at the left-bottom side of the area that will receive reports from the members of the formed coalitions. Without generality loss we fix $\lambda$ and $\mu$ in (3) as equal to 1. The m.D.M.C. performances are compared varying the sensor sensing range from $R_{S}=10 \mathrm{~m}$ to $R_{S}=30 \mathrm{~m}$. It is worth noting that assuming that no sensor can be placed at a distance of less than its own $R_{S}$ the following temperatures will be below growing $R_{S}$.

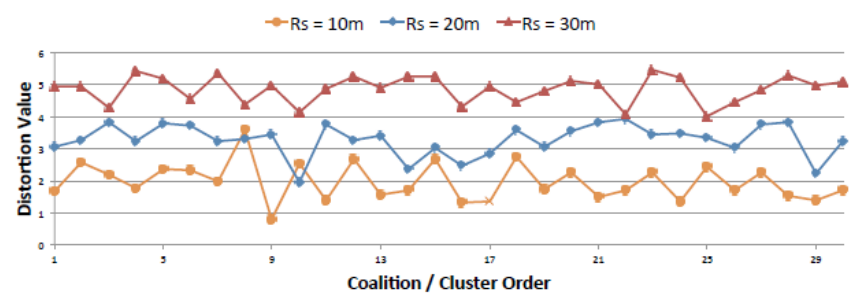

Fig. 3. Different trends of distortion function as the sensing range changes.

In Fig.3, we report the values of distortion function defined in (1). Here it is possible to note that high distortion values are not tied to the coalitions size. Instead, they are tied to the members characteristics and the following growth of the standard deviance which heavily influences the distortion function. Anyway, the protocol steadies distortion upon satisfactory values keeping distortion values in a smaller range.

\section{B. m.D.M.C. Vs. LNCA}

Here, we compare the performances of the m.D.M.C. protocol with those obtained using a LNCA [5] protocol on the same network topology of 225 gridded sensors. In LNCA, nodes first exchange information about their data readings, then, according to the similarity of data, form k-hop clusters, in our simulations $k=2$.

From the cluster form perspective the algorithm is a traditional k-hop clustering, but with random cluster sizes because of the data similarity requirement. In our simulations we intend to analyze the performance of those two protocols about the distortion reached.

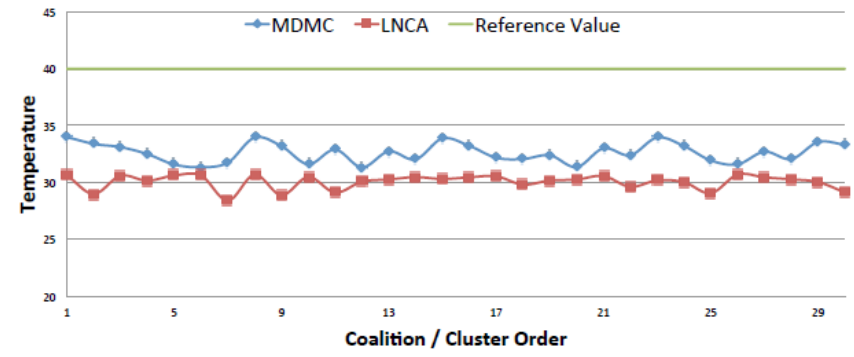

Fig. 4. Comparison among the temperature measurements reported to the sink.

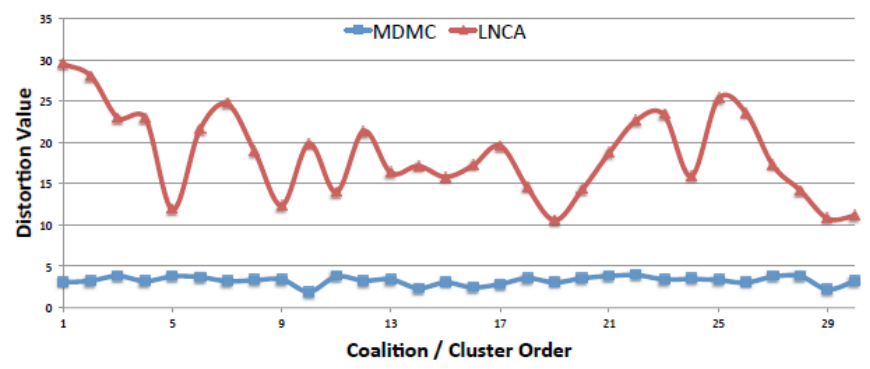

Fig. 5. Different trends of the distortion function tied to two protocols.

values, sent packets and spent energy. As above, we perform simulations that must guarantee thirty complete processes of either cluster head definition or coalition formation, and after average out the values.

1) Distortion Analysis: In the Fig.4 we show the temperature values at the sink computed using the reports sent by cluster head or coalition members. Comparing the trends with the reference value, it is easy to note how our protocol can reach estimates closer to the real value with an improvement on the entire LNCA performance. This better performance could just be justified by the m.D.M.C. distortion trend, shown in the Fig.5, but we think that it is also the result of a more uniform cover of the phenomenon produced by m.D.M.C..

2) Packet Analysis: In the Fig.6, we show the number of exchanged messages by m.D.M.C. protocol to complete a process of the coalition formation and the number of exchanged messages by LNCA to select all the cluster heads. Even now the messages sent by m.D.M.C. protocol are almost 25 percent less compared to those sent by LNCA protocol on the overall simulation. This, of course, results in a considerable saving in network traffic. 


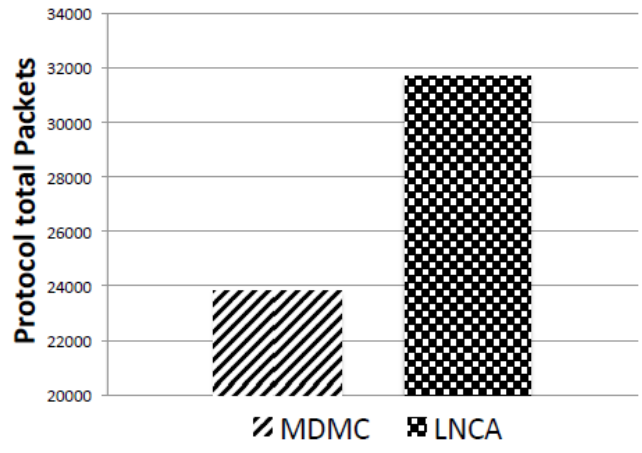

Fig. 6. Comparison between the number of packets which are sent by sensors to build the coalition or to select the cluster head.

3) Energy Analysis: Comparing the protocol energetic costs, we compute the energy spent by these protocols. Even though the m.D.M.C. protocol sends less messages than LNCA its energetic advantages decreases as shown in Fig.7. These m.D.M.C. lower performances are tied to the exchanged message size, indeed although m.D.M.C. sends less messages these ones are averagely heavier than ones sent by LNCA. Nevertheless this result may be further improved by extending the reporting phase. So we have shown, by using m.D.M.C. protocol that we can extend the life of the whole network, by reducing the number of exchanged messages and the energetic costs without worsening reliability.

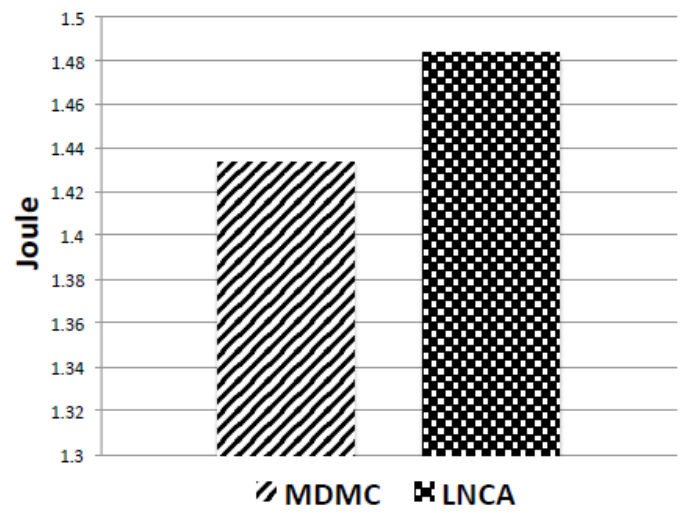

Fig. 7. Average energy spent by a node during the whole simulation.

\section{CONCLUSIONS}

This work applies the coalition process in a WSN environment. In the proposed coalition model, each sensor joins a coalition only if it realizes an advantage. Our cooperative model is divided into two steps: in the first step sensors bargain with one another about merging (or not) into a coalition, while in the second one sensors in one coalition negotiate about their payoff allocations. If the coalition is stable, sensors are satisfied with their payoffs and participate in the functions within the coalition. A distributed algorithm was also implemented to realize the coalition formation process inspired by recent works on the building of connected dominating sets. The parameters used in the algorithm allow us to maintain the distortion trend achieved by many coalitions in our simulations nearly constant. The simulation results confirm theoretical ones, guaranteeing reliable measurements at the sink. Our protocol also limits the number of exchanged messages, with an improvement of the performance of the whole sensors network. Nevertheless, our choices have been validate by the simulation results and it gives us a boost to further this research.

\section{REFERENCES}

[1] Akyildiz, Spatio-temporal correlation: theory and applications for wireless sensor networks, Computer Networks: The International Journal of Computer and Telecommunications Networking archive Volume 45, Issue 3 (June 2004), 245259.

[2] A. Forster, A.Forster, A.L.Murphy, Optimal cluster size for wireless sensor networks: An experimental analysis, Vol. 28, Part 1, 2010

[3] Nguyen, T.N., Duy, T.T., Tran, P.T., Voznak, M. Performance evaluation of user selection protocols in random networks with energy harvesting and hardware impairments (2016) Advances in Electrical and Electronic Engineering, 14 (4), pp. 372-377.

[4] Nguyen, H.-S., Do, D.-T., Voznak, M. Two-way relaying networks in green communications for 5G: Optimal throughput and tradeoff between relay distance on power splitting-based and time switching-based relaying SWIPT (2016) AEU - International Journal of Electronics and Communications, 70 (12), pp. 1637-1644.

[5] A. Taha, S. S. Soliman and A. Badawi, "Genetic algorithms for lifetime elongation of clustered WSN," 2017 IEEE 28th Annual International Symposium on Personal, Indoor, and Mobile Radio Communications (PIMRC), Montreal, QC, 2017, pp. 1-7. doi: 10.1109/PIMRC.2017.8292597

[6] M. Razzaq, D. Devi Ningombam and S. Shin, "Energy efficient K-means clustering-based routing protocol for WSN using optimal packet size," 2018 International Conference on Information Networking (ICOIN), Chiang Mai, Thailand, 2018, pp. 632-635. doi: 10.1109/ICOIN.2018.8343195

[7] Xia D., Vlajic N., Near-optimal node clustering in wireless sensor networks for environment monitoring., in Proc. of the 21st Int. Conf. on Advanced Networking and Applications, 2007.

[8] A. Forster, A.L. Murphy, CLIQUE: Role-free clustering with Q-learning for Wireless Sensor Networks, in Proc. of the 29st Int. Conf. on Distr.Computing (ICDCS), Canada, 2009

[9] A.W. Martin,S. Hussain, Intelligent hierarchical cluster-based routing, in Proc. of the 29st Int. Works. on Mobility and Scalability in Wireless Sensor Networks (MSWSN), San Francisco, CA, 2006

[10] N.Aslam, W.Philips, W.Robertson, A Unified Clustering and Communication Protocol for Wireless Sensor Networks, International Journal on Computer Science, 2008.

[11] X. Yang, Y. Yan and D. Deng, "Research on clustering routing algorithm based on K-means++ for WSN," 2017 6th International Conference on Computer Science and Network Technology (ICCSNT), Dalian, China, 2017, pp. 330-333.doi: 10.1109/ICCSNT.2017.8343712

[12] Liu, Z., B. Wang and L. Guo, A survey on connected dominating set construction algorithm for wireless sensor networks, Inform. Technol.J., 2010.

[13] Wackernagel, Multivariate Geostatistics. An Introduction with Applications, Springer-Verlag. ISBN 354060127 9,1995

[14] G. Kannan, T. Sree Renga Raja, Energy efficient distributed cluster head scheduling scheme for two tiered wireless sensor network, Egyptian Informatics Journal, Volume 16, Issue 2, 2015, Pages 167-174, ISSN 1110-8665, https://doi.org/10.1016/j.eij.2015.03.001.

[15] Xiang Min, Shi Wei-ren, Jiang Chang-jiang, Zhang Ying, Energy efficient clustering algorithm for maximizing lifetime of wireless sensor networks, AEU - International Journal of Electronics and Communications, Volume 64, Issue 4, 2010, Pages 289-298, ISSN 1434-8411, https://doi.org/10.1016/j.aeue.2009.01.004 
[16] N. Javaid, T.N. Qureshi, A.H. Khan, A. Iqbal, E. Akhtar, M. Ishfaq, EDDEEC: Enhanced Developed Distributed Energy-efficient Clustering for Heterogeneous Wireless Sensor Networks, Procedia Computer Science, Volume 19, 2013, Pages 914-919, ISSN 1877-0509, https://doi.org/10.1016/j.procs.2013.06.125.

[17] https://www.omnetpp.org/

[18] https://github.com/boulis/Castalia 